\title{
BOOKS
}

\section{Indispensible guide to Aboriginal people's health}

Healing Traditions: The Mental Health of Aboriginal Peoples in Canada

Laurence J. Kirmayer and Gail Guthrie

Valaskakis, editors

UBC Press; 2008. 528 pp \$39.95 paper

$\mathrm{T}$ This essay collection, intended as a resource for health practitioners, students, policymakers, educators and others, questions the effectiveness of current biomedical, Western approaches to Aboriginal mental health treatment. These essays assert the importance of self-determination, arguing that when indigenous peoples shape their own treatment programs in accordance with their cultures, traditions and history, efficacy is greatly enhanced.

These essays, written by academics, researchers and mental health practitioners, could arguably serve as not only a resource, but also a guide to understanding Aboriginal peoples' social experience and collective past. Its discussion of how history, culture and tradition affect mental health could reconfigure the treatment of mental illness for Aboriginal people.

It introduces readers to indigenous mental health issues and discusses causes and manifestations of mental illness, such as the high suicide rates and alcohol abuse found in some communities. It also provides examples of how communities have developed resilience to these problems and discusses traditional Aboriginal approaches to mental health.

Aboriginal writers in the collection include its coeditor, the late Gail

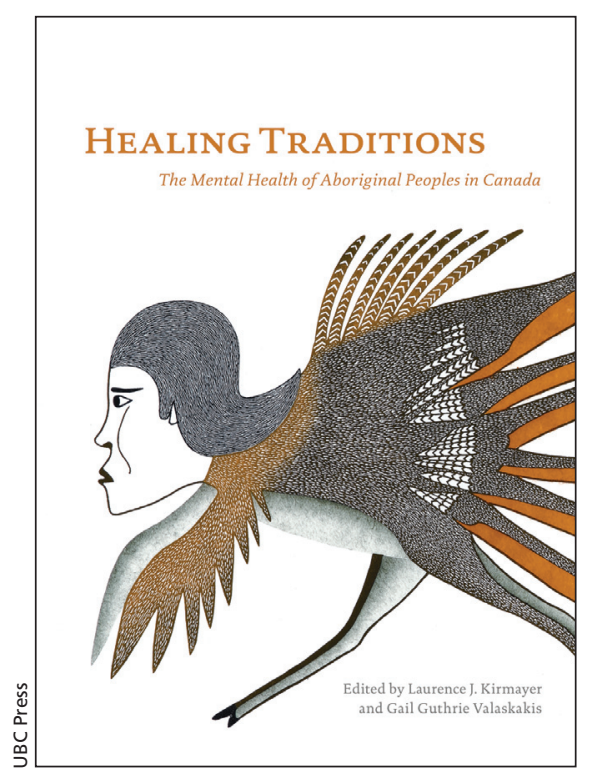

Valaskakis, the former director of research at the Aboriginal Healing Foundation. Cornelia Wieman, Canada's first Aboriginal psychiatrist, contributes an article about the Six Nations Reserve's unique approach to mental health services and Rod McCormick, a Mohawk and associate professor at the University of British Columbia, discusses approaches to psychological counselling grounded in Aboriginal perspectives.

Their essays and others call for a rethinking of Aboriginal mental health treatments. For example, a more effective modality involves an ecocentric approach to mental health, which recognizes how, for most Aboriginal people, hunting, fishing and trapping are deeply integrated with spiritual beliefs, family and community. A sociocentric view is also discussed as a remedy to the isolated individual, where the person being treated is seen as part of a web of relations that include family and community.

A positive example of the effect of self-determination is found in the Michael J. Chandler and Christopher E. Lalonde's essay: "Cultural Continuity as a Moderator of Suicide Risk among Canada's First Nations." They report that Aboriginal groups in British Columbia who have achieved a measure of self-determination have no youth suicides and low-to-zero rates of adult suicide (Transcult Psychiatry 1998; 35[2]:191-219). Self-determination includes self-government or involvement in land claims, community support for building cultural facilities and efforts to gain local control over health, education, policing and child welfare.

Given the need to address Aboriginal mental health problems nationally, and in a meaningful and effective way, Healing Traditions is timely and useful. Although aimed at a fairly general audience, this book's academic approach may limit its accessibility. However, for health professionals working with indigenous peoples, Healing Traditions may be an indispensible, philosophical and practical starting point.

\section{Jennifer Dales BA}

Writer, poet and reviewer Ottawa, Ont. 